\title{
Fast dose algorithm for generation of dose coverage probability for robustness analysis of fractionated radiotherapy
}

\author{
David Tilly $^{1,2)}$, Anders Ahnesjö ${ }^{1)}$ \\ 1) Medical radiation physics, department of immunology, genetics and pathology, Uppsala \\ University, Uppsala, Sweden \\ 2) Elekta Instruments $A B$, Uppsala, Sweden \\ E-mail: david.tilly@igp.uu.se
}

\begin{abstract}
A fast algorithm is constructed to facilitate dose calculation for a large number of randomly sampled treatment scenarios, each representing a possible realisation of a full treatment with geometric, fraction specific displacements for an arbitrary number of fractions. The algorithm is applied to construct a dose volume coverage probability map (DVCM) based on dose calculated for several hundred treatment scenarios to enable probabilistic evaluation of a treatment plan.
\end{abstract}

For each treatment scenario, the algorithm calculates the total dose by perturbing a precalculated dose, separately for the primary and scatter dose components, for the nominal conditions. The ratio of the scenario specific accumulated fluence, and the average fluence for infinite number of fractions is used to perturb the pre-calculated dose. Irregularities in the accumulated fluence may cause numerical instabilities in the ratio, which is mitigated by regularisation through convolution with a dose pencil kernel.

Compared to full dose calculations the algorithm demonstrates a speedup factor of $\sim 1000$. The comparisons to full calculations show $99 \%$ gamma index $(2 \% / 2 \mathrm{~mm})$ pass rate for a single highly modulated beam in a virtual water phantom subject to setup errors during five fractions. The gamma comparison show $100 \%$ pass rate in a moving tumour irradiated by a single beam in a lung-like virtual phantom. DVCM iso-probability lines computed with the fast algorithm, and with full dose calculations for each fraction, for a hypo-fractionated prostate case treated with rotational arc therapy treatment were almost indistinguishable. 


\section{Introduction}

The localisation uncertainties in fractionated radiotherapy are usually divided into a systematic component stemming from errors in the pretreatment imaging, and hence common to all treatment fractions, and a random component with a specific error at each fraction stemming from the daily patient setup procedures (van Herk et al 2000). By quantifying the uncertainties one can determine margin sizes as to create a planning target volume (PTV) for prescription such that certain probabilistic dose coverage goals are fulfilled. A common goal is that for $90 \%$ of the patients the clinical target volume (CTV) should receive at least $95 \%$ of the prescription dose (van Herk et al 2000).

An alternative strategy, commonly called probabilistic treatment planning, is to incorporate the dosimetric effects of geometrical uncertainties directly into the dose calculation and planning procedure and prescribe dose with explicit probability goals directly onto the CTV, thus eliminating the need for a margin based PTV (Baum et al 2006, Birkner et al 2003, Chan et al 2006, Li and Xing 2000, McShan et al 2006, Moore et al 2009, Rehbinder et al 2004, Sobotta et al 2010, Stroom et al 1999, Trofimov et al 2005, Witte et al 2007). Gordon et al (2010) suggested a framework for probabilistic treatment planning where the concept of CTV dose coverage was reused from the formalism of margin design for PTV based planning. By means of dose coverage probability they demonstrated that probabilistic plans yields better target dose coverage compared with margin based plans without compromising organ at risk doses for prostate patients. (Moore et al 2012, Bohoslavsky et al 2013) also used dose coverage probability to evaluate treatment plans generated with a probabilistic method versus plans based on margins, and showed that they could reduce the rectum dose while maintaining adequate target coverage. Despite successful proof of concept, practical implementation of probabilistic planning is hampered by long calculation times to generate the required dose coverage probability for the CTV.

Defining a treatment scenario as a realisation of a treatment with specifically sampled values for each fraction, the dose for a specific treatment scenario thus becomes the sum of the dose calculated for all fractions based on sampled values of the uncertainties. Given a priori distributions for the systematic and random uncertainties, a dose volume coverage map (DVCM) can thus be determined by scoring, for every simulated scenario, whether or not a given volume receives at least a certain dose (Gordon et al 2010). A DVCM is the full representation in the dose-volume domain of dose-population-

histograms (van Herk et al 2000, Gordon et al 2007). A sufficient amount of treatment scenarios must be simulated to achieve statistical confidence. Viewing dose coverage as a binomial probability distribution, i.e. either a coverage goal is met or not for a single scenario, almost 400 treatment scenarios will be needed to reach $\pm 3 \%$ accuracy $(95 \%$ CI) for a $90 \%$ coverage probability. Extending the framework to include also intra-fraction motion implies even more dose calculations. Hence, calculation of a DVCM can be computationally very demanding requiring development of specific algorithms for clinical implementation.

The effects of the random errors for infinite number of fractions can simply be estimated by convolving either the fluence (Beckham et al 2002), or the dose distribution (Leong 1987), with the displacement probability density function describing the random geometric errors. The results for treatments using only a few fractions can be significantly different (Craig et al 2003b). However, with explicit per fraction dose calculations based on randomised positional errors accurate dose coverage probabilities can be determined. Bohoslavsky et al (2013), Gordon et al (2010) and Moore et al (2012) all utilised shifting of the dose to estimate fraction specific doses, which in some cases may be sufficiently accurate but cannot accurately model the dose in situations with pronounced density heterogeneities (Craig et al 2003a). Even simple shifts of the dose distributions can be time consuming 
when simulating many hundred patient treatment scenarios as exemplified by Moore $e t$ al where a probabilistic optimisation with 128 scenarios took almost two days. Moreover, integration (e.g. through discretisation) over a time dependent dose is required to handle intra-fraction motion and thus cannot be handled by shifting of the total dose. Recent algorithmic and hardware development have reduced the dose calculation time down to $\sim 1$ s per plan for algorithms accelerated by graphics processing unit (GPU) (Xun et al 2014). However, even at this speed it will take $>30$ min to perform the 2000 dose calculations involved in a five fraction hypo-fractionated treatment for 400 treatment scenarios. Furthermore, for a full implementation of probabilistic planning, calculation of the involved DVCM needs to be integrated into the treatment plan optimisation process requiring a substantial number of optimisation iterations for which the calculations has to be repeated.

To alleviate some of the calculation burden, and to facilitate implementation of planning tools based on dose coverage probability, we present a fast dose calculation algorithm to enables efficient integration of dose affecting geometrical uncertainties into the planning process. The algorithm is based on perturbations of a pre-calculated dose distribution in order to accurately handle motion in heterogeneous situations such as lung. The incorporation of geometric uncertainties is general enough to include both systematic and random errors stemming from setup errors, inter- and intra-fraction motion, segmentation uncertainty and to some degree patient deformations. We will first investigate the dosimetric accuracy of the proposed algorithm for treatment scenarios in virtual phantoms, and then apply it to calculate dose coverage probabilities for a clinical prostate case treated with rotational therapy (VMAT) where we explicitly consider setup errors and prostate motion.

\section{Theory}

To calculate the dose for a treatment scenario, specific values for the geometrical errors at each fraction must be given. The uncertainty model used for this purpose is described in the first section below, followed by a description of the fast algorithm used for dose calculation of the treatment scenario.

\subsection{Sampling of geometric errors for a treatment scenario}

For the $i$ :th realisation of a treatment scenario, the total dose delivered after $N_{\mathrm{f}}$ fractions at the position $\boldsymbol{r}=(x, y, z)$ fixed in the patient anatomy, can be expressed as

$$
D^{i}(\boldsymbol{r})=\sum_{j=1}^{N_{\mathrm{f}}} d^{i, j}\left(\boldsymbol{r} ; \boldsymbol{\delta}^{i, j}\right)
$$

where $d^{i, j}$ is the dose for the $j$ :th fraction of the $i$ :th treatment scenario, and $\boldsymbol{\delta}^{i, j}$ is the geometric error sampled for a specific scenario and fraction. The geometric error is defined as the difference between the nominal position of an anatomy related point $\boldsymbol{r}$ and its corresponding, scenario and fraction specific position $\boldsymbol{r}^{i, j}$,

$$
\boldsymbol{\delta}^{i, j}=\left(\delta_{x}^{i, j}, \delta_{y}^{i, j}, \delta_{z}^{i, j}\right)=\boldsymbol{r}^{i, j}-\boldsymbol{r} .
$$

Generally, the geometrical error is location dependent such that $\boldsymbol{\delta}^{i, j}=\boldsymbol{\delta}^{i, j}(\boldsymbol{r})$, which would include deformations, but we will for simplicity assume $\boldsymbol{\delta}^{i, j}$ to be independent of position, i.e. rigid body translations with no rotations. The framework does not require $\boldsymbol{\delta}^{i, j}$ to be normally distributed, i.e. any reasonably well behaved distribution can be used.

The geometric deviation $\boldsymbol{\delta}^{i, j}$ is usually viewed as being composed of a systematic and a random component, where the systematic component arises from the treatment preparation process (van Herk 
et al 2000). Thus, for every simulated treatment scenario a systematic error $\boldsymbol{\delta}_{\mathrm{S}}^{i}$ is sampled and kept constant for all fractions in the scenario, and for each of the fractions in the scenario a random error $\boldsymbol{\delta}_{\mathrm{R}}^{i, j}$ is sampled such that

$$
\boldsymbol{\delta}^{i, j}=\boldsymbol{\delta}_{\mathrm{S}}^{i}+\boldsymbol{\delta}_{\mathrm{R}}^{i, j}
$$

It is straightforward to extend the framework to include intra-fraction motion, e.g. a lung tumour moving intra-fractionally along a trajectory. To implement that, the fraction dose $d^{i, j}$ can be discretised into $K$ samples in time such that

$$
D^{i}(\boldsymbol{r})=\sum_{j=1}^{N_{\mathrm{f}}} \sum_{k=1}^{K} d^{i, j, k}\left(\boldsymbol{r} ; \boldsymbol{\delta}^{i, j, k}\right)
$$

where $\boldsymbol{\delta}^{i, j, k}$ now becomes the geometric error for the $k$ :th time sample for the $j$ :th fraction of the $i$ :th treatment scenario. In equation (4), all contributions are for simplicity treated as equally weighted, i.e. representative for a treatment delivery with equal fluence weight per term.

\subsection{Fast calculation of the dose from a treatment scenario with fraction specific sampled uncertainties}

The main idea behind the fast dose algorithm is to approximate dose perturbations as proportional to perturbations in the incident energy fluence as seen by the targeted tissue. Such conditions are well suited for construction of a correction factor approach given that, 1) a similar pre-calculated dose distribution with non-zero dose values for an "unperturbed" geometry can be provided for all dose points of interest, 2) a corresponding energy fluence distribution exists with non-zero values, and finally 3 ) the energy fluence perturbations relative to the unperturbed situation can be determined by simple and fast means. The nominal fluence for the beam configuration is essentially zero outside the nominal field borders, making it unsuitable for use as the fluence for dose pre-calculation since the fluence perturbation factor cannot be calculated (division by zero). However, the average fluence for infinite number of fractions is simple and fast to calculate by convolving the nominal fluence with the uncertainty probability distribution. The dose distribution corresponding to this average fluence is also simple to calculate by any dose engine that accepts an incident fluence distribution as input. Hence, as basis for the correction factor approach we choose the average energy fluence for an infinite number of fractions $\Psi^{\infty}$, and its corresponding dose $D^{\infty}$. Figure 1 shows a flowchart of the algorithm as applied to the calculation of the doses from multiple treatment scenarios.

As the primary and scatter dose components (Ahnesjö and Aspradakis 1999) have different characteristics, and by most dose engines can be calculated separately, we treat them separately such that the estimate $\widetilde{D}^{i}(\boldsymbol{r})$ of the dose for treatment scenario $i$ is given by

$$
\widetilde{D}^{i}(\boldsymbol{r})=\widetilde{D}_{\mathrm{p}}^{i}(\boldsymbol{r})+\widetilde{D}_{\mathrm{s}}^{i}(\boldsymbol{r})
$$

where the primary and scatter parts are estimated by

$$
\widetilde{D}_{\mathrm{p}}^{i}(\boldsymbol{r})=\mathcal{C}_{\mathrm{p}}^{i}(\boldsymbol{r}) \cdot D_{\mathrm{p}}^{\infty}(\boldsymbol{r})
$$

and

$$
\widetilde{D}_{\mathrm{s}}^{i}(\boldsymbol{r})=\mathcal{C}_{\mathrm{s}}^{i}(\boldsymbol{r}) \cdot D_{\mathrm{s}}^{\infty}(\boldsymbol{r}),
$$

respectively, with separate correction factors $\mathcal{C}_{\mathrm{p}}^{i}$ and $\mathcal{C}_{\mathrm{s}}^{i}$ applied to the pre-calculated primary and scatter components, $D_{\mathrm{p}}^{\infty}$ and $D_{\mathrm{s}}^{\infty}$, of the pre-calculated total dose $D^{\infty}$. Any suitable, accurate dose 
engine that can deliver the primary and scatter dose can be used to calculate $D_{\mathrm{p}}^{\infty}$ and $D_{\mathrm{s}}^{\infty}$. In this work we use the pencil kernel (Ahnesjö et al 1992) or the collapsed cone (Ahnesjö 1989) algorithm implemented in the TPS (Oncentra ${ }^{\circledR} 4.3$ ) (Ahnesjö et al 2005) but e.g. a Monte Carlo dose engine could be used as well. The procedures to estimate $\mathcal{C}_{\mathrm{p}}^{i}$ and $\mathcal{C}_{\mathrm{s}}^{i}$ are similar so only $\mathcal{C}_{\mathrm{p}}^{i}$ will in the following be explicitly described in detail.

The average of the sampled fluence per fraction exiting the treatment machine, as seen from a coordinate system fixed in the patient with the beam direction along the $z$ axis, is for the $i$ :th treatment scenario given by

$$
\Psi^{i}(x, y)=\frac{1}{N_{\mathrm{f}}} \sum_{j}^{N_{\mathrm{f}}} \Psi\left(x-\delta_{x}^{i, j}, y-\delta_{y}^{i, j}\right)
$$

where $\Psi(x, y)$ is the nominal fluence. The average fluence from an infinite number of scenarios $N_{\mathrm{s}}$ and fractions is thus

$$
\Psi^{\infty}(x, y)=\lim _{\substack{N_{\mathrm{f}} \rightarrow \infty \\ N_{\mathrm{s}} \rightarrow \infty}} \frac{1}{N_{\mathrm{s}}} \frac{1}{N_{\mathrm{f}}} \sum_{i}^{N_{\mathrm{s}}} \sum_{j}^{N_{\mathrm{f}}} \Psi\left(x-\delta_{x}^{i, j}, y-\delta_{y}^{i, j}\right)
$$

which can be calculated by convolving $\Psi(x, y)$ with the part of the distribution for $\boldsymbol{\delta}^{i, j}$ that is lateral to the beam direction. The dose components $D_{\mathrm{p}}^{\infty}$ and $D_{\mathrm{s}}^{\infty}$ are calculated using $\Psi^{\infty}$ as input. The correction factors, $\mathcal{C}_{\mathrm{p}}^{i}$ and $\mathcal{C}_{\mathrm{s}}^{i}$, could in principle be estimated by the ratio of $\Psi^{i}$ to $\Psi^{\infty}$. However, the dose is also affected by the depth component of $\boldsymbol{\delta}^{i, j}$ and to include this the fluence for each fraction is weighted with the longitudinal change in attenuation and inverse square law to yield the effective sampled fluence

$$
\widetilde{\Psi}_{\mathrm{p}}^{i}\left(x, y ; z_{0}\right)=\frac{1}{N_{\mathrm{f}}} \sum_{j}^{N_{\mathrm{f}}} \Psi\left(x-\delta_{x}^{i, j}, y-\delta_{y}^{i, j}\right) \frac{d_{\mathrm{p}}^{z}\left(z_{\mathrm{rad}}\left(z_{0}+\delta_{z}^{i, j}\right)\right)}{d_{\mathrm{p}}^{z}\left(z_{\mathrm{rad}}\left(z_{0}\right)\right)}\left(\frac{S}{S+\delta_{z}^{i, j}}\right)^{2},
$$

where $d_{\mathrm{p}}^{z}$ is the depth dose (in water) for the primary component, $z_{\mathrm{rad}}(z)$ is the radiological depth, and $z_{0}$ is a chosen representative geometrical depth, and $S$ is the nominal distance from the source to $z_{0}$. The depth correction in equation (10) require in general a new raytrace to determine $z_{\mathrm{rad}}\left(z_{0}+\right.$ $\delta_{z}^{i, j}$ ) due to potential organ motion introduced by the sampled error. However, in the interest of calculation speed we assume that the average linear attenuation coefficient does not change so that we can simply scale $z_{\text {rad }}$ with the change in geometrical depth $\delta_{z}^{i, j}$. If needed an additional raytrace could be employed for a more accurate depth correction. The effective fluence for the scatter dose component, $\widetilde{\Psi}_{\mathrm{s}}^{i}$, differs only from $\widetilde{\Psi}_{\mathrm{p}}^{i}$ because the depth doses, $d_{\mathrm{p}}^{z}$ and $d_{\mathrm{s}}^{z}$, are different. Intra-fraction motion can be included by introducing a sum over time samples in equation (10) analogously to equation (4).

Before the calculated fluences are used to determine correction factors for the dose, both $\widetilde{\Psi}_{\mathrm{p}}^{i}$ and $\Psi^{\infty}$ are convolved with a primary dose pencil kernel, $\frac{p_{\mathrm{p}}}{\rho}$, defined at a representative depth $z_{0}$. This makes the perturbation process more robust since the pencil kernel convolution acts as a regularisation where irregularities in $\widetilde{\Psi}_{\mathrm{p}}^{i}$, occurring especially for hypo-fractionated treatments, will to some degree be washed out due to the low pass filtering properties of the fluence to dose conversion. Effectively this 
means that the perturbations are introduced via the dose domain, which is smoother than the fluence domain. Finally, the primary dose perturbation factor is given by

$$
\mathcal{C}_{\mathrm{p}}^{i}(\boldsymbol{r})=\frac{\widetilde{\Psi}_{\mathrm{p}}^{i}(x, y) \otimes \frac{p_{\mathrm{p}}}{\rho}}{\Psi^{\infty}(x, y) \otimes \frac{p_{\mathrm{p}}}{\rho}}
$$

The correction factor for the scattered component,

$$
\mathcal{C}_{\mathrm{s}}^{i}(\boldsymbol{r})=\frac{\widetilde{\Psi}_{\mathrm{s}}^{i}(x, y) \otimes \frac{p_{\mathrm{s}}}{\rho}}{\Psi^{\infty}(x, y) \otimes \frac{p_{\mathrm{s}}^{\prime}}{\rho}}
$$

is calculated analogously but with scatter data for the depth dose correction in the effective fluence, $\widetilde{\Psi}_{\mathrm{S}}^{i}$, and a scatter dose pencil kernel, $\frac{p_{\mathrm{s}}}{\rho}$, in the regularisation step.

The sampled accumulated fluence, $\widetilde{\Psi}_{\mathrm{p}}^{i}$, is only valid for those body sites which motion pattern can be described by the same probability distribution used in the fluence accumulation since we assumed rigid body motion. Therefore, $\widetilde{\Psi}_{\mathrm{p}}^{i}$ must be calculated separately for region of interests (ROI) with different motion pattern. However, the extra computational burden is limited since the pre-calculated distributions $\Psi^{\infty} \otimes \frac{p}{\rho}, D_{\mathrm{p}}^{\infty}$ and $D_{\mathrm{s}}^{\infty}$ can be reused as long as the current geometry does not differ too much.

The achieved calculation efficiency arises from the fact that the fraction by fraction accumulation (including the pencil kernel convolution) is made using the 2D fluence distributions instead of the 3D dose distributions. The speedup, as compared to a brute force full calculation where all fraction doses are calculated separately, will be greater for treatment schemes with more fractions. The effort of calculating the treatment scenario dose to a point is reduced to two scalings and one summation, i.e. equations (5), (11) and (12) since $\Psi^{\infty} \otimes \frac{p}{\rho}, D_{\mathrm{p}}^{\infty}$ and $D_{\mathrm{s}}^{\infty}$ can all be pre-calculated. The algorithm consists of simple branchless operations on large blocks of data, which makes it a good candidate for effective implementation on parallelised hardware such as GPUs. 


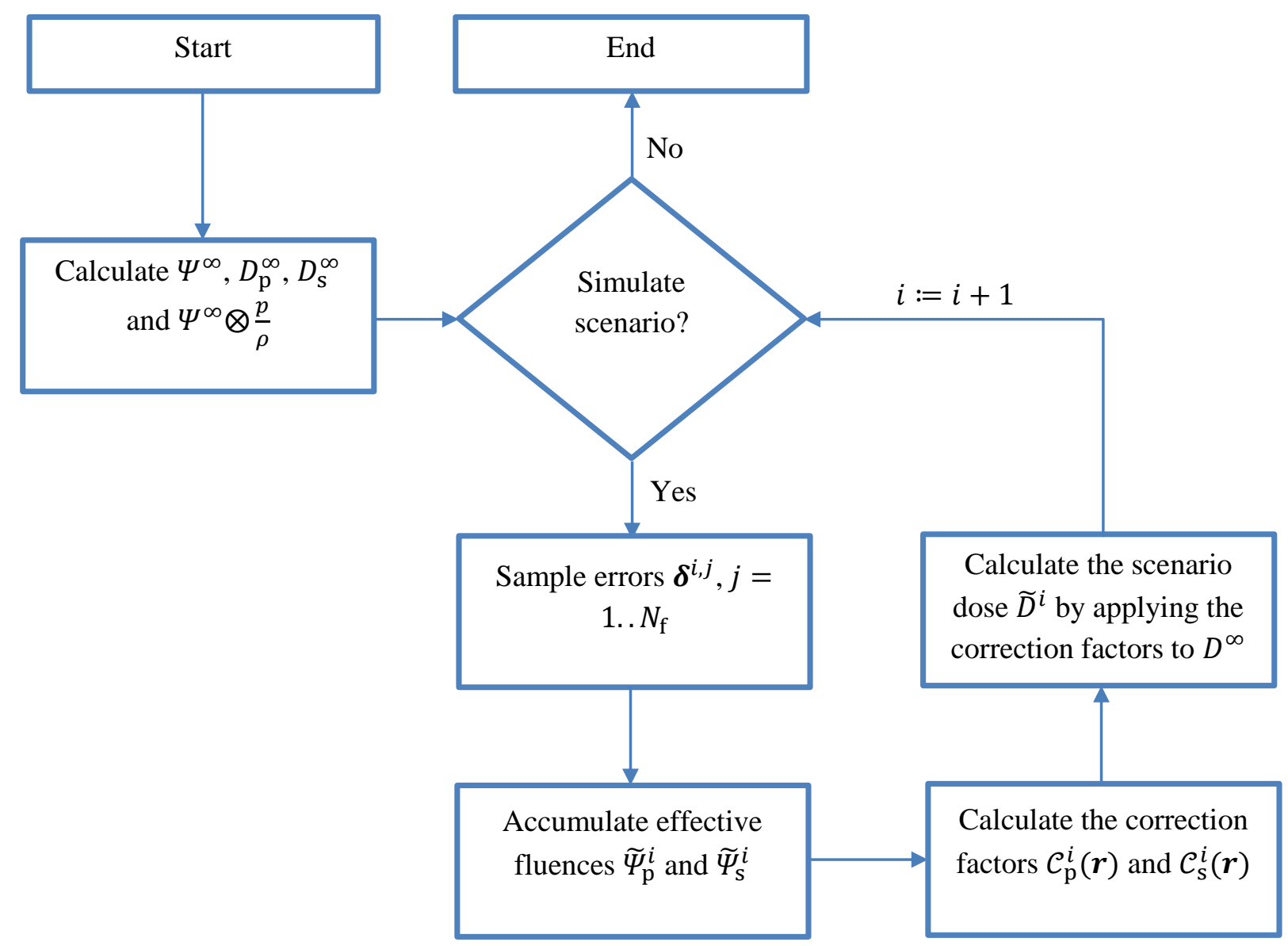

Figure 1. Flowchart of the perturbation algorithm for calculation of dose distributions for treatment scenarios.

\section{Methods}

The fast algorithm was tested by comparing treatment scenario doses for selected cases. The first case was a highly modulated beam aimed at a homogeneous water phantom and subject to setup errors. The aim of the more challenging second test was to investigate if the accuracy of the algorithm was good enough to be used for dose calculation of lung tumour treatments. Moreover, it also demonstrated the simulation of intra-fraction organ motion. We calculated the dose for different tumour locations as well as the total fraction dose for a tumour moving along an elliptical trajectory in a lung-like heterogenous phantom. Finally, the generation and utilisation of DVCMs is demonstrated for a prostate patient. The result from the perturbation algorithm was in all three cases compared to full calculations where an explicit dose calculation for each geometry with sampled position displacements was made, as in equation (1), using the same dose engine as for the pre-calculated dose, i.e. pencil kernel algorithm for the water case and prostate case, and the collapsed cone algorithm for the lunglike case. Dosimetric evaluation was performed using the gamma comparison (Low et al 1998) $(2 \% / 2 \mathrm{~mm})$ using the speed improvements described by (Wendling et al 2007).

\subsection{A single modulated beam in a water phantom}

A single $6 \mathrm{MV}$ beam from a highly modulated IMRT treatment was selected as representative for testing. A source to surface distance of $90 \mathrm{~cm}$ was chosen. The distributions $D_{\mathrm{p}}^{\infty}$ and $D_{\mathrm{s}}^{\infty}$ were calculated using the pencil kernel method (Ahnesjö et al 1992). Twenty treatment scenarios were generated for a five fraction (5fx) regimen with setup errors sampled from a 3D isotropic normal distribution (standard deviations of $0.5 \mathrm{~cm}$ along the coordinate axes, with all covariances equal to zero). The estimated treatment scenario doses $\widetilde{D}^{i}$ were compared to results from full calculations using 
the pencil kernel method for each of the individual fraction doses where the isocenter was moved according to the same samples of the fraction specific errors.

\subsection{A single beam in a lung-tumour phantom.}

A single beam was placed with its axis perpendicular to the surface of a lung-like phantom with the isocenter located at the center of the tumour in the nominal position, see figure $2 \mathrm{a}$. The phantom consisted of a $3 \mathrm{~cm}$ thick water slab covering the lung tissue (ICRU 1989) with density $0.30 \mathrm{~g} / \mathrm{cm}^{3}$. A spherical tumour consisting of water with a $2 \mathrm{~cm}$ diameter was placed at $10 \mathrm{~cm}$ depth. The intrafraction motion of lung tissue can exhibit complex trajectories (Low et al 2005). In this work we used an elliptical tumour trajectory with minor axis $2.0 \mathrm{~mm}$ and major axis $7.5 \mathrm{~mm}$, which was laid out in two different ways to create two different trajectories, $T_{1}$ and $T_{2}$. This will mimic motion relative the beam axis for two gantry angles separated by $90^{\circ}$ with trajectory $T_{1}$ defined with the major axis parallel to the beam axis, see figure $2 \mathrm{~b}$, and $T_{2}$ with the major axis lateral in the field (perpendicular to the beam axis). Both trajectories were discretised in 11 evenly spread out tumour locations, $T_{1}^{1, \ldots, 11}$ and $T_{2}^{1, \ldots, 11}$ respectively. For each trajectory a beam was conformed to a hypothetical PTV defined by a $5 \mathrm{~mm}$ margin around the internal target volume (ITV) formed by the union of all space occupied by the tumour sphere moving along the trajectory.

First, for each individual location in the trajectory the result for the perturbation algorithm was compared to collapsed cone calculations, where the tumour successively was located to the different locations in the trajectory. If the perturbation algorithm is accurate for all $T_{1}^{1, \ldots, 11}$ and $T_{2}^{1, \ldots, 11}$ we assume that it would be accurate also for more complex trajectories.

Secondly, the total dose for the entire trajectory was calculated by summing over all $T_{1}^{1, \ldots, 11}$ (and $T_{2}^{1, \ldots, 11}$ respectively) in equation (10) (the sum over fractions is removed since we calculate the dose of a single fraction). Again, results for the perturbation algorithm were compared to full calculations using the collapsed cone algorithm.

In treatment planning many different beam configurations are normally tried, either manually or by an iterative optimisation procedure, until a treatment plan with the prescribed CTV dose coverage is obtained. The comparison between the perturbation algorithm and the collapsed cone was therefore repeated for the trajectory $T_{2}$ using a beam configuration conformed to only partially cover the ITV as to stress testing under less symmetrical conditions as symmetry may cause error cancelation. The beam was chosen to conform to an ITV of a trajectory with only half the radii of $T_{2}$.

As described in equation (9), the average fluence, $\Psi^{\infty}$, can be calculated by convolution of the incident fluence with the uncertainty probability distribution. However, the trajectory of the lung tumour is not easily described by a distribution. Empirically it was found that a normal distribution with $0.3 \mathrm{~cm}$ standard deviation (and zero covariances) in all directions yielded accurate dosimetric results. The narrower beam configurations were somewhat sensitive to the size of the standard deviation where a too small standard deviation can cause numerical instability in the scaling procedure of equations (11) and (12). 


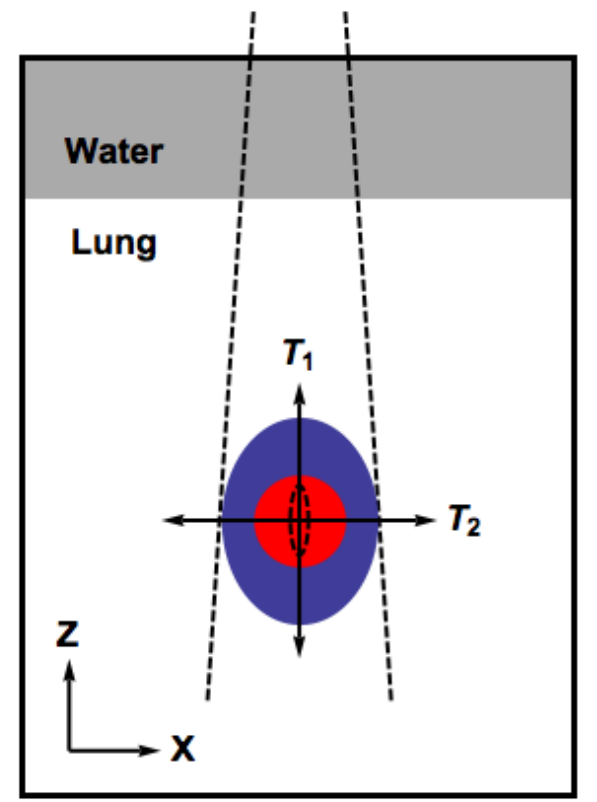

(a)

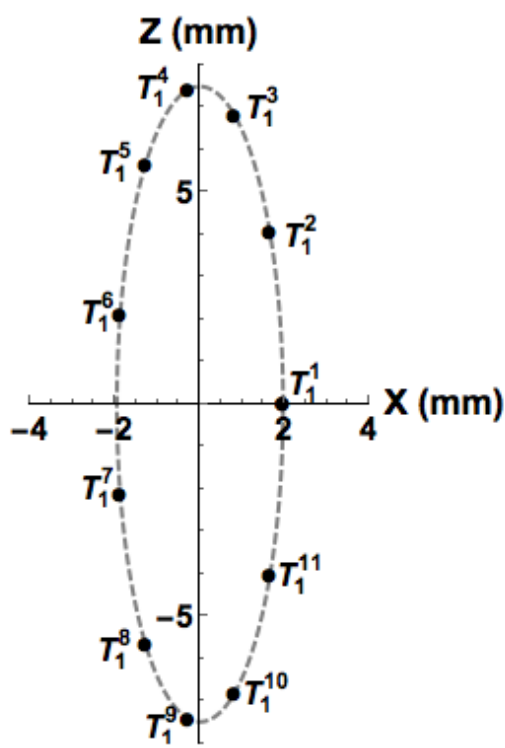

(b)

Figure 2. (a) A tumour (red) at $10 \mathrm{~cm}$ depth located with its center $7 \mathrm{~cm}$ inside lung tissue covered by $3 \mathrm{~cm}$ water. The direction of the major axis of the trajectories $T_{1}$ (dashed ellipse) and $T_{2}$ are indicated by the arrows. The incident beam (dashed) is conformed to the PTV (ITV $+5 \mathrm{~mm}$ margin) (blue) based on $T_{1}$ and $T_{2}$ respectively. (b) The trajectory $T_{1}$ and its the discretisation into the locations $T_{1}^{1, \ldots, 11}$.

\subsection{The dose coverage probability for a prostate patient}

The calculation of dose coverage probability was tested with data based on a representative prostate patient. A PTV was constructed based on the margin recipe by van Herk et al (2000), using the uncertainties in table 1, to ensure that the entire CTV (prostate) receives a minimum of $95 \%$ of the prescribed dose for $90 \%$ of the treatment scenarios resulting in 7, 14 and $14 \mathrm{~mm}$ margins in the L-R, S-I and A-P directions, respectively. A $360^{\circ}$ single arc VMAT plan (hypo-fractionated, $5 \mathrm{fx}$ to $35 \mathrm{~Gy}$ ) was created according to the planning strategy specified in table 2 using the Oncentra ${ }^{\circledR}$ TPS where an objective with higher priority was given a higher weight in the optimisation. The plan robustness for the prostate CTV, rectum and bladder was investigated by creating a DVCM, as described by Gordon et al (2010), based on 400 treatment scenarios each with fraction specific errors sampled from a normal distribution with the standard deviations (zero covariances) as given in table 1 . The prostate was considered to move as a rigid body between fractions (without changing the surrounding tissue) and the rectum and bladder were considered to be subject only to setup errors. The iso-probabilities in the DVCM based on the perturbation algorithm was compared to iso-probabilities based on the full calculation using the pencil kernel method where the setup error was handled as isocenter shifts and the prostate motion again as a rigid body translation.

Table 1. The geometric uncertainties in terms of standard deviations (SD) used for the prostate case.

\begin{tabular}{lcccccc}
\hline & \multicolumn{3}{c}{ Systematic errors (mm) } & \multicolumn{3}{c}{ Random errors (mm) } \\
& L-R & S-I & A-P & L-R & S-I & A-P \\
\hline Organ motion (Byrne 2005) & 1.0 & 4.0 & 4.0 & 1.0 & 4.0 & 4.0 \\
Setup error (van Herk 2004) & 2.0 & 2.0 & 2.0 & 2.0 & 2.0 & 2.0 \\
Total SD & 2.2 & 4.5 & 4.5 & 2.2 & 4.5 & 4.5
\end{tabular}


Table 2. Optimisation criteria used for treatment planning of the prostate case. The doses in the objectives are relative the prescription dose $35 \mathrm{~Gy}$.

\begin{tabular}{lcc}
\hline \multicolumn{1}{c}{ ROI } & Objective & Priority \\
\hline PTV & $D_{98 \%}>95 \%$ & 1 \\
& $D_{2 \%}<105 \%$ & 2 \\
Normal Tissue & 95\%-50\% dose fall-off in $2 \mathrm{~cm}$ from & 3 \\
& PTV & \\
Rectum & Minimise $V_{31.4 G y}$ & 4 \\
Bladder & $V_{33.7 \mathrm{~Gy}}<25 \%$ & \\
& $V_{31.4 \mathrm{~Gy}}<35 \%$ & 5 \\
& $V_{29.2 \mathrm{~Gy}}<50 \%$ & 5 \\
\hline
\end{tabular}

\section{Results}

\subsection{Dosimetric accuracy of treatment scenario doses for a single field in a water phantom} Dose profiles at $10 \mathrm{~cm}$ depth intersecting the beam axis for a scenario with a substantially different dose compared to the static dose $D_{0}$ (no setup errors) are shown in figure 3 . The agreement between $\widetilde{D}$ and $D$ is quantified by the 3D gamma $(2 \% / 2 \mathrm{~mm})$ index $\gamma$. Only points with a dose higher than $2 \%$ of the max dose was included in the comparison. The $2 \%$ dosimetric criteria is relative the max dose of the treatment scenario. All simulated scenarios exhibited comparable accuracy and the combined cumulative distribution, see figure $4 \mathrm{a}$, shows that more than $99 \%$ of all tested points from all 20 scenarios passed the $(2 \% / 2 \mathrm{~mm})$ criteria. By analyzing the combined gamma distribution separately per depth, see figure $4 \mathrm{~b}$, it was clear that the majority of points violating the gamma criteria were located at the surface (depth $\leq 2 \mathrm{~cm}$ ) where $6 \%$ of the tested points violated the gamma criteria whereas virtually no points at all violated the criteria at the chosen reference depth $\left(z_{0}=10 \mathrm{~cm}\right)$. The pencil kernel is defined at a representative depth $z_{0}$ and the further away the depth is from $z_{0}$, the larger the error since the kernel data changes. The pencil kernel changes most near the surface and that is the reason for the asymmetry around $z_{0}=10 \mathrm{~cm}$ in figure $4 \mathrm{~b}$.

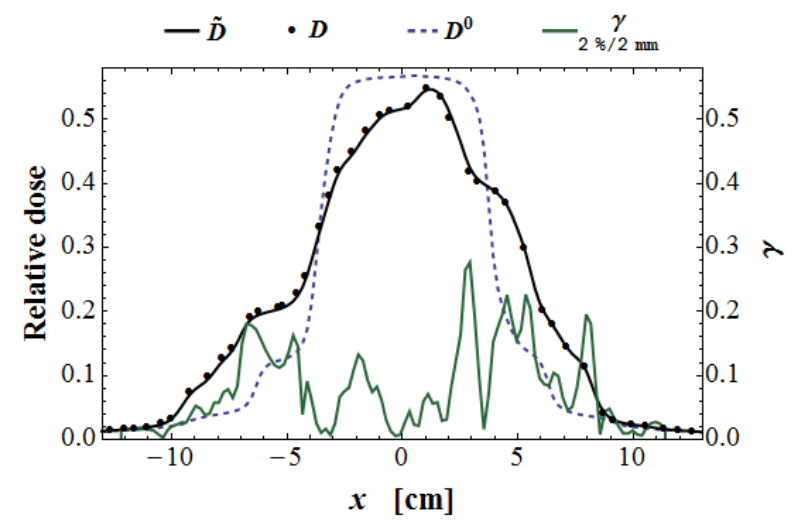

(a)

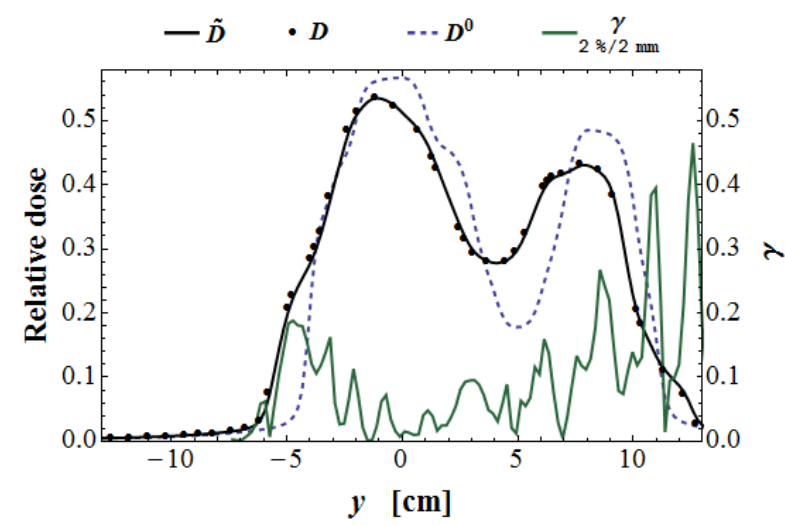

(b)

Figure 3. Dose profiles (a) parallel $(x)$ and (b) perpendicular $(y)$ to the leaf direction at $10 \mathrm{~cm}$ depth for one of the $5 \mathrm{fx}$ treatment scenarios, produced by a single modulated IMRT beam and fraction specific setup errors. The dose was normalised to the dose for calibration conditions $\left(10 \times 10 \mathrm{~cm}^{2}\right.$ beam, 
$\mathrm{SSD}=90 \mathrm{~cm}, 10 \mathrm{~cm}$ depth). Results for the perturbation algorithm, $\widetilde{D}$, (solid black) are shown with full dose calculation, $D$, (dots black) and compared using the 3D gamma comparison (solid green). The static dose profiles without setup error, $D^{0}$, (dashed blue) is shown for comparison.

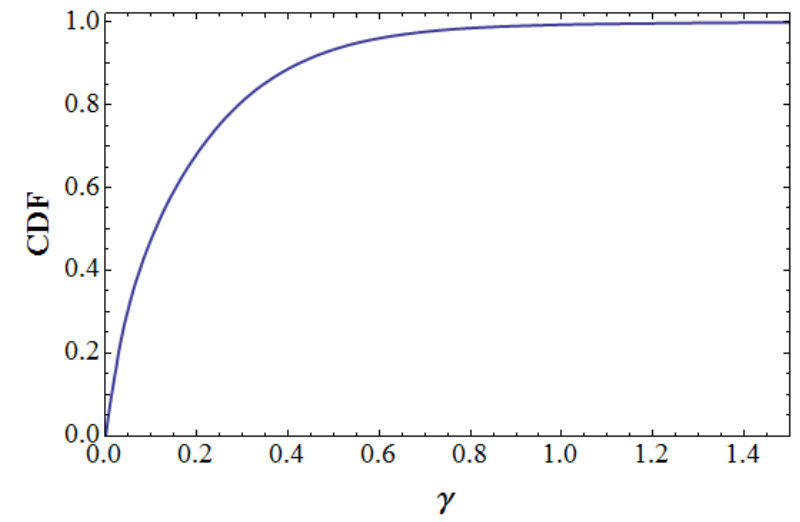

(a)

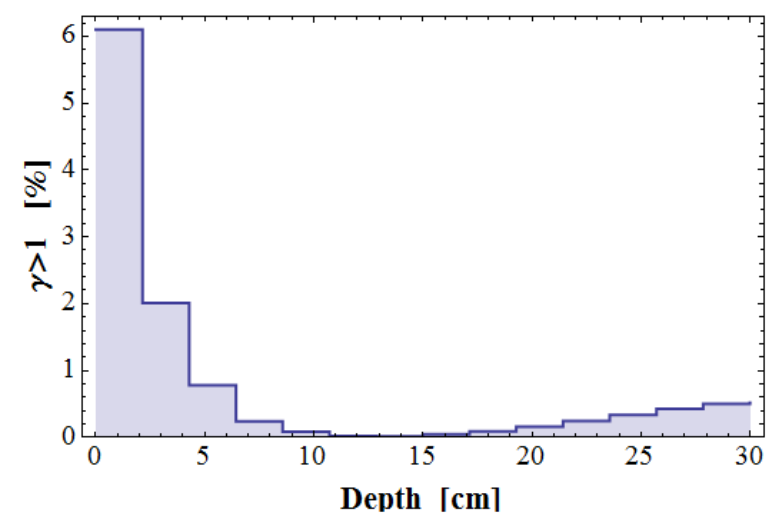

(b)

Figure 4. (a) The combined cumulative gamma distribution for all simulated $5 \mathrm{fx}$ treatment scenarios for the single IMRT beam onto a water phantom. (b) The percentage of points per depth interval from all scenarios failing the $2 \% / 2 \mathrm{~mm}$ criteria.

\subsection{Dosimetric accuracy for a single field in a lung-like phantom with a moving tumour}

The aim of the more challenging test using a lung phantom was to investigate if the accuracy of the algorithm was good enough to be used for dose calculation of lung tumour treatments. Moreover, it also demonstrated the simulation of intra-fraction organ motion, i.e. a lung tumor moving along elliptical trajectories as specified in figure 2.

The dose was calculated using the perturbation algorithm for all tumour individual locations along the trajectories as well as for the entire trajectories. Dose profiles through the center of the tumor are shown in figure 5 together with the 3D gamma comparison $(2 \% / 2 \mathrm{~mm}$ where the dosimetric criteria was relative the max dose in the lung tumor) with the collapsed cone full dose calculation. The accuracy was comparable for all evaluated $T_{1}^{1, \ldots, 11}$ and $T_{2}^{1, \ldots, 11}$. The combined cumulative gamma distributions based on all dose points in the tumour, from $T_{1}^{1, \ldots, 11}$ and $T_{2}^{1, \ldots, 11}$ respectively, are shown in figure 6a. Not a single dose point in the entire tumour from any of the tumour locations violated the gamma criteria. The cumulative gamma distributions for the total dose of the trajectories $T_{1}$ and $T_{2}$ are shown in figure 6 .

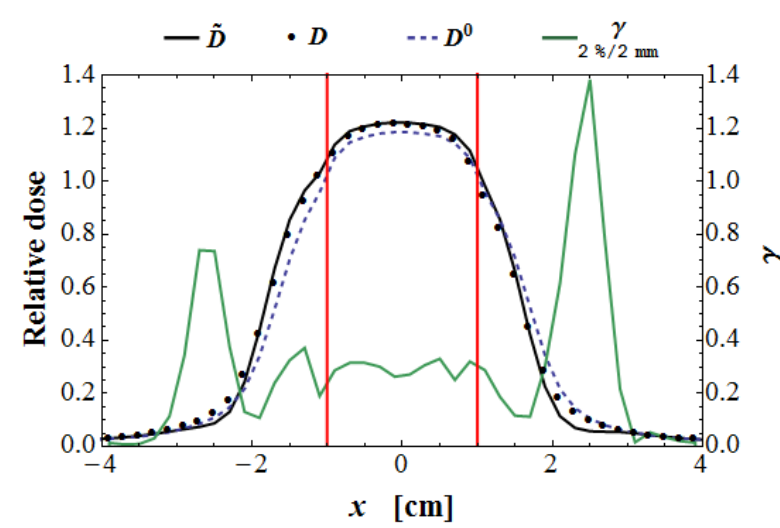

(a)

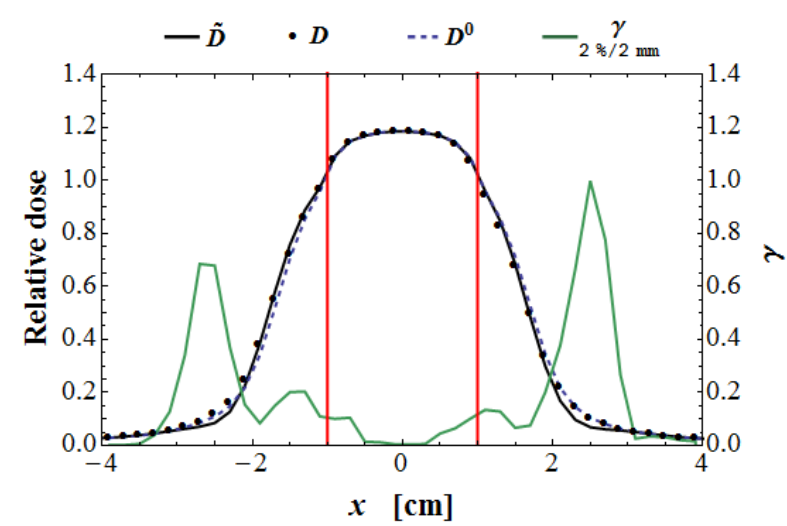

(b)

Figure 5. Dose profiles parallel to the leaf direction $(x)$ through the center of the tumor for (a) the trajectory position $T_{2}^{11}$ and (b) the total trajectory $T_{2}$. The dose was normalised to the calibration dose 
(cf. figure 4). Results for the perturbation algorithm, $\widetilde{D}$, (solid black) was compared with full calculations done with the collapsed cone method, $D$, (dots black) using the $3 \mathrm{D}$ gamma comparison (solid green). The tumour CTV border is shown by the vertical lines (red). The static dose profiles without organ motion (dashed blue) are shown for comparison.

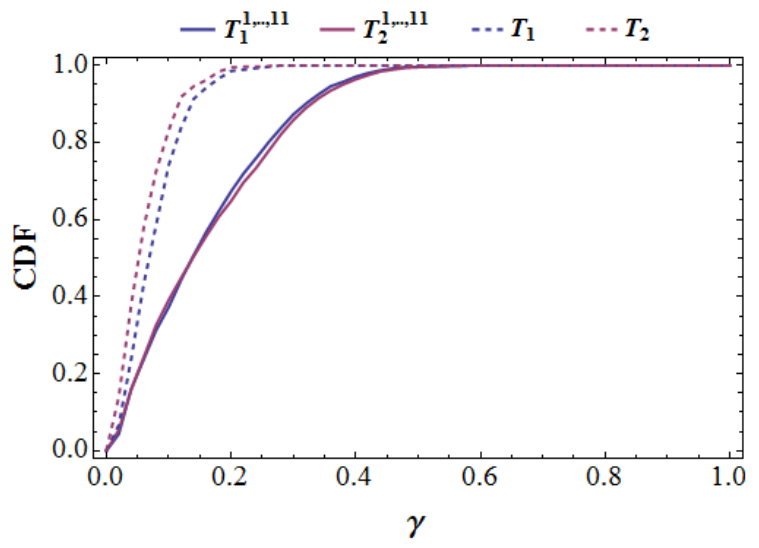

(a)

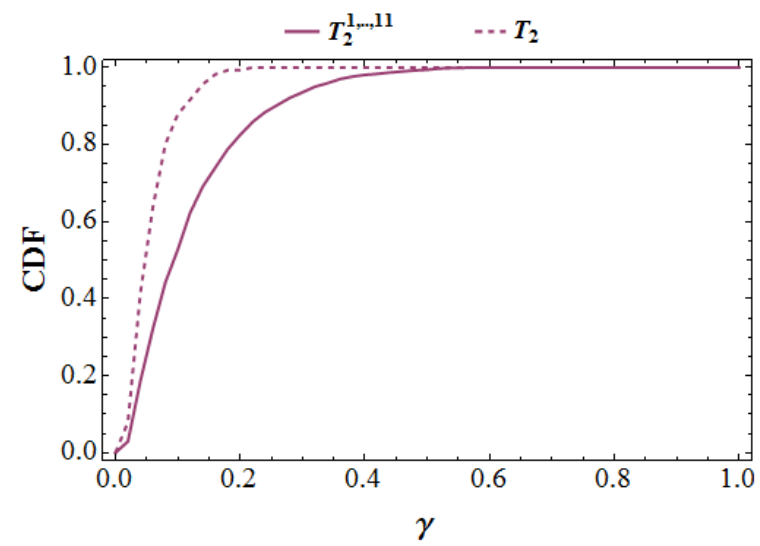

(b)

Figure 6. The combined cumulative gamma $(2 \% / 2 \mathrm{~mm})$ distribution for all trajectory positions $T_{1}^{1, \ldots, 11}$ and $T_{2}^{1, \ldots, 11}$ individually (solid), and for the case where total trajectory dose was calculated directly (dashed), using a single beam (a) conformed to the PTV (ITV $+5 \mathrm{~mm}$ margin) and (b) only partially covering the ITV. Only dose points belonging to the tumor were included in the analysis.

The same analysis was repeated for the trajectory $T_{2}$ using the beam only partially covering the ITV again using the collapsed cone as comparison. Dose profiles through the center of the tumour for a single trajectory position as well as for the entire trajectory are shown in figure 7. Again, not a single point in the entire tumour violated the gamma criteria. The cumulative gamma distributions, based on all dose points in the tumour, for all $T_{2}^{1, \ldots, 11}$ combined and for the total dose of the trajectory $T_{2}$ are shown in figure $6 b$.

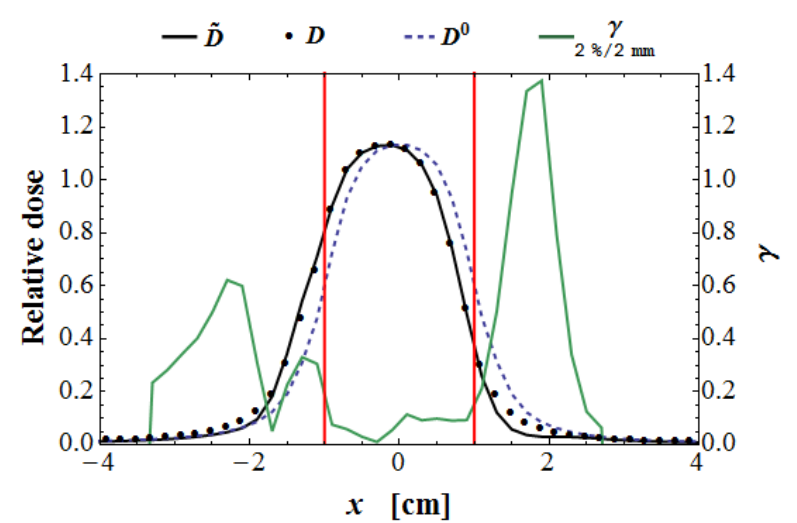

(a)

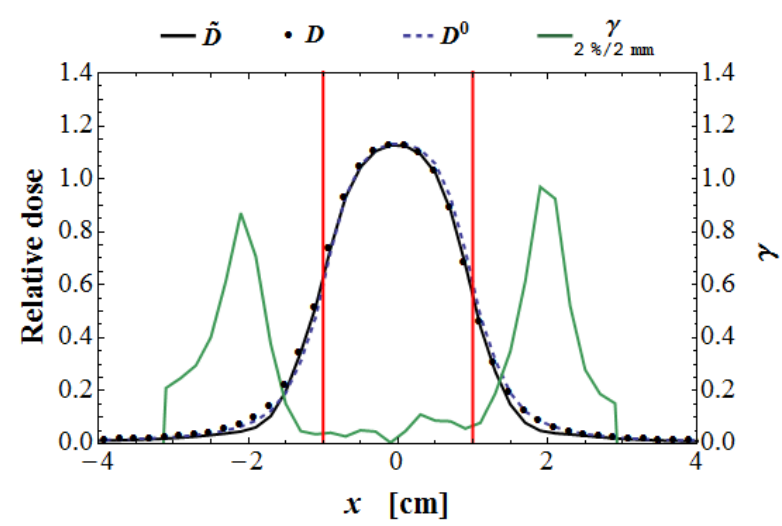

(b)

Figure 7. Dose profiles parallel to the leaf direction $(x)$ through the center of the tumor for (a) the trajectory position $T_{2}^{1}$ and (b) the total trajectory $T_{2}$ for a beam conformed to only partially cover the ITV. The dose was normalised to the calibration dose (cf. figure 4). Results for the perturbation algorithm, $\widetilde{D}$, (solid black) was compared with the collapsed cone, $D$, (dots black) dose calculation using the 3D gamma comparison (solid green). The tumour CTV border is shown by the vertical lines (red). The static dose profiles without organ motion (dashed blue) are shown for comparison. 


\subsection{Dose coverage probability for a prostate case}

The proposed fast dose algorithm was applied to a prostate case and 400 treatment scenarios were simulated so that a DVCM could be calculated based on the resulting DVHs for the prostate CTV, rectum and bladder. A subset of these DVHs are shown in figure 8a to illustrate the agreement between the perturbation algorithm and the reference calculation. The DVHs for all the 400 simulated treatment scenarios are shown in figure $8 \mathrm{~b}$ along with the $90 \%$ iso-probability lines extracted from the DVCMs based on the perturbation algorithm and using full calculations. The result of the perturbation algorithm is in this context indistinguishable from the full calculations.

The iso-probability lines of a DVCM can be used as an evaluation metric in margin free dose planning. The isoline shows the partial volume that receives at least a certain dose level for a chosen probability (here 90\%) and can be used to evaluate the plan robustness. Common margin recipe aim to fulfill $95 \%$ of the prescribed dose to the entire CTV for $90 \%$ of the treatment scenarios and thus we can evaluate the CTV dose coverage by comparing the $90 \%$ probability isoline versus the $95 \%$ of the prescribed dose. A closer inspection of the $90 \%$ probability isoline in figure $8 \mathrm{~b}$ shows that the entire prostate will receive at least $95 \%$ of the prescribed dose. The $10 \%$ iso-probability is shown for the organ at risks, i.e. the volume that receives at most a certain dose in $90 \%$ of the treatment scenarios.

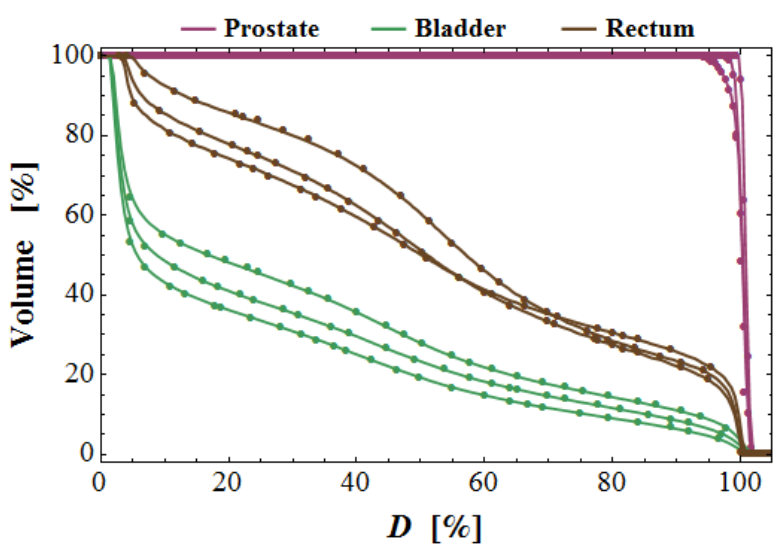

(a)

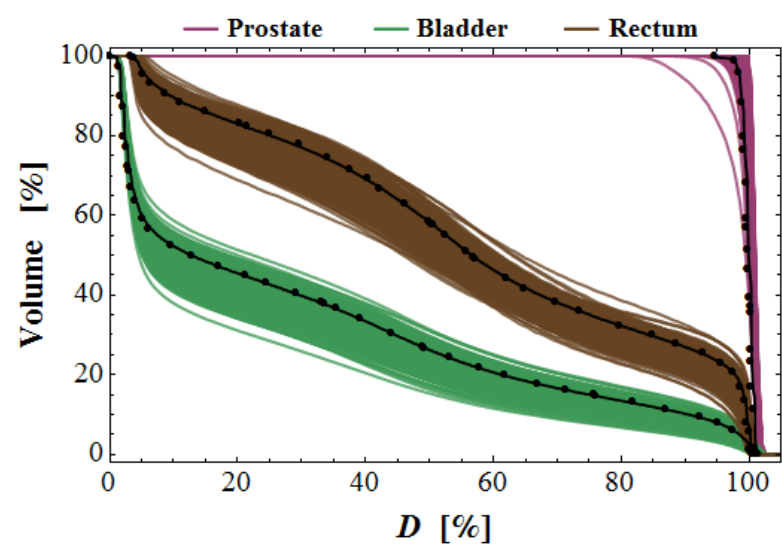

(b)

Figure 8. (a) DVH comparison between the reference calculation (dots) and the perturbation algorithm (solid lines) for three different treatment scenarios. (b) The DVHs for 400 sampled scenarios with the resulting $90 \%$ probability isoline (black) in the dose coverage probability map. The iso-probability lines based on full calculations (dots) and the perturbation algorithm (solid) are practically indistinguishable.

\subsection{Calculation speed}

The proposed algorithm, implemented on a Intel Xeon E5-2620 CPU, 6 cores @ $2.0 \mathrm{GHz}, 16 \mathrm{~GB}$ RAM was able to calculate dose for the $5 \mathrm{fx}$ in $3.2 \cdot 10^{7}$ points $/ \mathrm{sec}$ per beam direction (single thread) once the pre-calculation (120s for 90 beam directions in the VMAT case) of $D^{\infty}$ was done. The algorithm can easily be multithreaded over the dose points, however, for a CPU implementation it is more efficient to use a threading model where each scenario is run in sequence in one thread and the different scenarios are spread over different threads. A near linear speedup with the number of cores was achieved once the pre-calculation had been performed.

The time to calculate (multi-threaded) the 400 scenarios for the prostate VMAT treatment was $\sim 7$ minutes which yields a speedup of over 1000 times compared to the full pencil kernel calculations. The speedup versus collapsed cone would be greater since the proposed fast algorithm stays the same, except that $D^{\infty}$ is calculated with collapsed cone, whereas the full dose calculations would involve a 
vast amount of collapsed cone calculations. A 64bit operating system (and program) was required and benefitted from 16 GB RAM to keep the $D^{\infty}$ from all 90 beam directions in memory. The speedup for an arbitrary number of scenarios was estimated by timing of the different parts of the calculation and then scaling, if appropriate, with the number of scenarios. Because of the initial pre-calculation of $D^{\infty}$ the speedup will increase with more scenarios, or if more fractions were used per scenario, or if intrafraction motion would be included, see figure 9. The difference in calculating the scenario dose using the fast algorithm for $5 \mathrm{fx}$ or $35 \mathrm{fx}$ was a factor of $\sim 2.5$ due to the extra fluence superposition (c.f. equation (10)). A higher resolution of the fluence map (2 $\mathrm{mm})$ or dose grid $(2 \mathrm{~mm})$ would increase the execution time. The VMAT dose calculations in this work, both the full calculation and using the proposed algorithm, are done by discretising the beam arc in 90 directions. The speedup is therefore expected to be similar for a smaller arc than $360^{\circ}$ or a treatment using a finite number of beams.

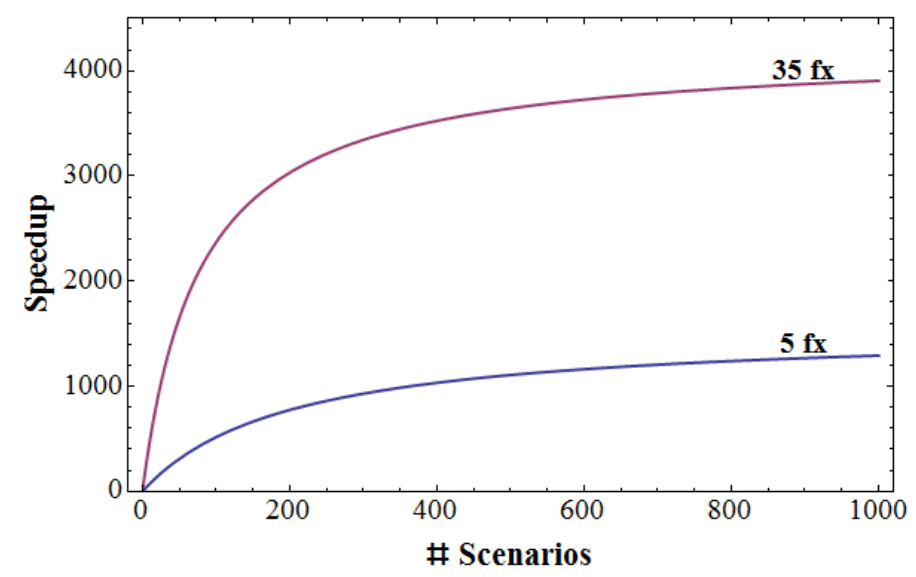

Figure 9. The speedup factor of the proposed algorithm compared to full calculations for a VMAT prostate treatment.

\section{Discussion}

The accuracy of the proposed algorithm is very promising. The phantom tests show that a $2 \% / 2 \mathrm{~mm}$ accuracy is possible for the proposed algorithm in the total dose for entire treatment scenarios, both in a water phantom but also in a lung like phantom with a small $(2 \mathrm{~cm}$ diameter) moving tumor. This could make the algorithm suitable for several applications in radiotherapy planning such as dose coverage calculations, as exemplified, but also for other purposes such as dose estimates in optimisation loops. The speed of the algorithm should make use of dose coverage probability as a clinical evaluation tool practical. The proposed algorithm is well suited for implementation on massive paralleled hardware, such as a GPU, since it calculates the dose for each dose point separately by a relatively simple scaling procedure.

The dose algorithm perturbs a pre-calculated dose distribution, $D^{\infty}$, which implies that the dose accuracy is limited by the accuracy of the dose engine used for $D^{\infty}$. In heterogeneous situations, such as in lung, a sophisticated algorithm like collapsed cone or Monte Carlo must hence be used as the pre-calculation dose engine. To avoid numerical instabilities in the fluence ratio scaling, c.f. equations (11) and (12), care should be taken so that the uncertainty distribution used in the calculation of the average fluence, $\Psi^{\infty}$, is not too narrow.

It can be noted that the static dose calculated for the nominal tumour position was a good approximation of the total trajectory dose for this particular trajectory, see figure $5 \mathrm{~b}$ and $7 \mathrm{~b}$. However, Figure $5 \mathrm{a}$ and $7 \mathrm{a}$ indicate that this might not be true for all possible trajectories and in those cases the perturbation algorithm could be more accurate than the static dose. 
We have shown that both random and systematic error (setup error and rigid organ motion) can be included for calculation of treatment scenario doses. There are other uncertainties such as segmentation uncertainties, CT to material lookup etcetera, which should be included in the design of a treatment plan. Segmentation uncertainty can be included through the use of realistic scenario specific segmentation samples. Deformations can be handled in the same way as long as the dose distribution does not change too much due to the deformation. A very explicit way to incorporate deformation would be to allow position dependent uncertainties to be merged into the fluence accumulation procedure. This could possibly be done by use of the projection of the 3D displacement vector field (produced by the deformable registration) averaged along beam ray lines to the representative depth for each beam. In principle, this could also be discriminated with respect to different anatomical volumes with different movement patterns and hence radiation exposure.

The algorithm can at present be directly used as an evaluation tool. Further development is needed to incorporate the algorithm into a robust optimisation scheme where the objective function uses dose coverage probabilities.

\section{Conclusions}

A fast dose algorithm to calculate the total dose from an entire treatment was developed for the purpose of evaluating a treatment plan in a margin less planning paradigm. The dosimetric agreement with full calculations is sufficient with calculation speed enough to enable integration of dose coverage probability evaluations into a clinical workflow.

\section{Acknowledgements}

The authors would like to thank Virgil Willcut for reviewing the manuscript.

\section{References}

Ahnesjö A 1989 Collapsed cone convolution of radiant energy for photon dose calculation in heterogeneous media Med Phys 16 577-92

Ahnesjö A and Aspradakis M M 1999 Dose calculations for external photon beams in radiotherapy Phys Med Biol 44 R99-155

Ahnesjö A, Saxner M and Trepp A 1992 A pencil beam model for photon dose calculation Med Phys $19263-73$

Ahnesjö A, Weber L, Murman A, Saxner M, Thorslund I and Traneus E 2005 Beam modeling and verification of a photon beam multisource model Med Phys 32 1722-37

Baum C, Alber M, Birkner M and Nusslin F 2006 Robust treatment planning for intensity modulated radiotherapy of prostate cancer based on coverage probabilities Radiother. Oncol. 78 27-35

Beckham W A, Keall P J and Siebers J V 2002 A fluence-convolution method to calculate radiation therapy dose distributions that incorporate random set-up error Phys Med Biol 47 3465-73

Birkner M, Yan D, Alber M, Liang J and Nusslin F 2003 Adapting inverse planning to patient and organ geometrical variation: algorithm and implementation Med. Phys. 30 2822-31

Bohoslavsky R, Witte M G, Janssen T M and van Herk M 2013 Probabilistic objective functions for margin-less IMRT planning Phys. Med. Biol. 583563 
Byrne T E 2005 A review of prostate motion with considerations for the treatment of prostate cancer Med. Dosim. 30 155-61

Chan T C Y, Bortfeld T and Tsitsiklis J N 2006 A robust approach to IMRT optimization Phys. Med. Biol. 51 2567-83

Craig T, Battista $\mathbf{J}$ and Van Dyk $\mathbf{J}$ 2003a Limitations of a convolution method for modeling geometric uncertainties in radiation therapy. I. The effect of shift invariance Med Phys 30 2001-11

Craig T, Battista $\mathbf{J}$ and Van Dyk $\mathbf{J} 2003 \mathrm{~b}$ Limitations of a convolution method for modeling geometric uncertainties in radiation therapy. II. The effect of a finite number of fractions Med Phys $\mathbf{3 0}$ 2012-20

Gordon J J, Sayah N, Weiss E and Siebers J V 2010 Coverage optimized planning: probabilistic treatment planning based on dose coverage histogram criteria. Med. Phys. 37 550-63

Van Herk M 2004 Errors and margins in radiotherapy Semin Radiat Oncol 14 52-64

Van Herk M, Remeijer P, Rasch C and Lebesque J V 2000 The probability of correct target dosage: dose-population histograms for deriving treatment margins in radiotherapy Int J Radiat Oncol Biol Phys 47 1121-35

ICRU 1989 Tissue Substitutes in Radiation Dosimetry and Measurement vol 44

Leong J 1987 Implementation of random positioning error in computerised radiation treatment planning systems as a result of fractionation Phys Med Biol 32 327-34

Li J G and Xing L 2000 Inverse planning incorporating organ motion Med. Phys. 27 1573-8

Low D A, Harms W B, Mutic S and Purdy J A 1998 A technique for the quantitative evaluation of dose distributions Med Phys 25 656-61

Low D A, Parikh P J, Lu W, Dempsey J F, Wahab S H, Hubenschmidt J P, Nystrom M M, Handoko M and Bradley J D 2005 Novel breathing motion model for radiotherapy Int J Radiat Oncol Biol Phys 63 921-9

McShan D L, Kessler M L, Vineberg K and Fraass B A 2006 Inverse plan optimization accounting for random geometric uncertainties with a multiple instance geometry approximation (MIGA) Med. Phys. 33 1510-21

Moore J A, Gordon J J, Anscher M S and Siebers J V 2009 Comparisons of treatment optimization directly incorporating random patient setup uncertainty with a margin-based approach $\mathrm{Med}$. Phys. 36 3880-90

Moore J A, Gordon J J, Anscher M, Silva J and Siebers J V 2012 Comparisons of treatment optimization directly incorporating systematic patient setup uncertainty with a margin-based approach Med. Phys. 39 1102-11

Rehbinder H, Forsgren C and Lof J 2004 Adaptive radiation therapy for compensation of errors in patient setup and treatment delivery Med. Phys. 31 3363-71

Sobotta B, Sohn M and Alber M 2010 Robust optimization based upon statistical theory Med. Phys. 37 4019-28 
Stroom J C, de Boer H C J, Huizenga H and Visser A G 1999 Inclusion of geometrical uncertainties in radiotherapy treatment planning by means of coverage probability Int. J. Radiat. Oncol. Biol. Phys. 43 905-19

Trofimov A, Rietzel E, Lu H M, Martin B, Jiang S, Chen G T Y and Bortfeld T 2005 Temporo-spatial IMRT optimization: concepts, implementation and initial results Phys. Med. Biol. 50 2779-98

Wendling M, Zijp L J, McDermott L N, Smit E J, Sonke J J, Mijnheer B J and van Herk M 2007 A fast algorithm for gamma evaluation in 3D Med Phys 34 1647-54

Witte M G, van der Geer J, Schneider C and Lebesque J V 2007 IMRT optimization including random and systematic geometric errors based on the expectation of TCP and NTCP Med. Phys. 34 $3544-55$

Xun J, Peter Z and Steve B J 2014 GPU-based high-performance computing for radiation therapy Phys. Med. Biol. 59 R151 